\title{
FUSIÓN DE SERIES TEMPORALES DE NUBES DE PUNTOS PARA SU EXPLORACIÓN EN REALIDAD VIRTUAL
}

\author{
Samuel Punzón Agudo, Emiliano Pérez Hernández, María Pilar Merchán García, Santiago Salamanca Miño, \\ Alejandro Espacio Regalado \\ Escuela de Ingenierías Industriales. Universidad de Extremadura. Avda. de Elvas, s/n. 06006 Badajoz, \\ \{spunzon, emilianoph, pmerchan, ssalamanca, alespacior\}@unex.es
}

\begin{abstract}
Resumen
Las nubes de puntos cada vez pueden contener más información, parte de la cual puede ser variable en el tiempo. Por este motivo, es valioso poder generar nubes que contengan la información correspondiente a diferentes instantes de tiempo sin la redundancia de información espacial. De esta forma, se consigue que el procesamiento y la visualización de los datos sea más sencillo, lo que beneficia a sistemas que necesitan que la información que manejen esté optimizada, como son los sistemas de Realidad Virtual. Por lo tanto, se propone una solución que mediante la fusión de las nubes de puntos tomadas en distintos instantes genera una nube resultado con la información óptima. Como prueba de funcionamiento se ha creado una aplicación de Realidad Virtual en la que se permite la exploración y sondeo de la información de la nube.
\end{abstract}

Palabras clave: nubes de puntos, Realidad Virtual, información térmica, optimización de la información, visualización de información.

\section{INTRODUCCIÓN}

Hoy en día existen múltiples técnicas para escanear espacios tridimensionales como las tratadas en [1]. Estas tecnologías permiten obtener información muy precisa de las dimensiones del espacio en formato de nubes de puntos. Con el tiempo, la mayoría de los dispositivos de digitalización han ido incorporando cámaras que permiten añadir información de color a la nube de puntos. Además, en la actualidad es posible capturar nubes de puntos con otras propiedades, bien porque algunos fabricantes hayan desarrollado nuevos dispositivos con más sensores incorporados o bien porque algunos investigadores hayan incorporado nuevos elementos al sistema de digitalización, como el trabajo mostrado en [2].

En ocasiones las propiedades que se miden, para una misma ubicación del escáner, varían con el tiempo, registrándose diferentes valores dependiendo de la hora del día o de la estación en la que se produzca la captura de la información. Por lo que se podría diferenciar entre propiedades estáticas, como son las coordenadas 3D, y propiedades dinámicas, variables en el tiempo. En este sentido, puede resultar de interés el poder representar el valor de alguna propiedad dinámica en diferentes instantes de tiempo. Para ello, es necesario diseñar un procedimiento para el registro de las nubes de puntos adquiridas en instantes de tiempo diferentes en una única nube que permita recuperar el valor de tal propiedad en los diferentes momentos en que fueran adquiridos.

Por otro lado, con la mejora de las prestaciones de los escáneres $3 \mathrm{D}$, el volumen de datos que generan ha ido siendo cada vez mayor. A esto se añade el hecho de que la utilización de múltiples nubes de puntos tomadas en la misma ubicación provoca que haya un número muy elevado de puntos a representar. Esto conlleva que, de cara al rendimiento en la visualización, exploración e interacción con las nubes de puntos puede resultar costoso gestionar tanta información. Esto puede ser especialmente significativo en los sistemas de Realidad Virtual donde la optimización ha de ser un paso clave, debido a la gran demanda de potencia gráfica de estos sistemas.

En este artículo se propondrá una solución que permita resolver dicha problemática y se diseñará una aplicación de Realidad Virtual que se beneficie de la optimización conseguida, probando de esta forma la utilidad del sistema. Para el desarrollo de la aplicación se utilizará el motor de videojuegos Unity, ya que otros autores [3] han demostrado su utilidad en el trabajo con nubes de puntos.

El artículo se estructura como sigue: en el apartado 2 se describe el mecanismo de fusión de nubes de puntos capturadas en diferentes instantes de tiempo. En el apartado 3 se trata la aplicación de Realidad Virtual desarrollada para probar la exploración y sondeo de la nube de puntos resultado. Por último, en el apartado 4, se desarrollan las conclusiones y perspectivas de futuro del trabajo.

\section{MÉTODO DESARROLLADO}

El método que se propone en este artículo parte de un conjunto de nubes de puntos, en adelante "nubes en 
bruto", cuyos puntos tienen información de una propiedad estática: coordenadas tridimensionales y de una propiedad dinámica, que, en concreto, se ha elegido que sea la temperatura capturada para cada punto. Estas nubes son obtenidas tras la digitalización de una de las estancias del interior de un edificio, mediante la ubicación del escáner en las posiciones que cubran todas las superficies de la estancia y repitiendo la adquisición en diferentes instantes.

El objetivo del método es obtener una nube de puntos en la que pueda visualizarse la evolución de la propiedad dinámica en una aplicación de Realidad Virtual.

El método sigue el proceso que se esquematiza en la Figura 1, y que se explica en las secciones siguientes.

\subsection{REGISTRO}

El primer paso que se ha de llevar a cabo es el alineamiento y registro de esas nubes en un mismo sistema de referencia. Se trata de un registro atemporal y redundante, en el sentido de que se han de colocar en el mismo sistema de coordenadas las nubes obtenidas en todas las adquisiciones, tanto las realizadas secuencialmente para cubrir la adquisición de la estancia entera, como las realizadas en otros instantes de tiempo, que pueden distar horas o incluso días, de las capturas anteriores, pero que tendrán cierta redundancia de información de coordenadas $3 \mathrm{D}$ con respecto a las anteriores.

El problema del registro, aunque viene facilitado por la asistencia que ofrece el software propio del escáner $3 \mathrm{D}$, requiere de la aplicación de algoritmos tales como el clásico "Iterative Closest Point" [4], ICP, o algunas de sus variantes [5].

Como ejemplo se muestran, en las Figuras 2.a), 2.b), 2.c) y 2.d), las nubes en bruto \#1, \#2, \#3 y \#4, respectivamente, que se han adquirido en los instantes de tiempo $t_{1}, t_{2}, t_{3}$ y $t_{4}$, donde $t_{1}$ y $t_{2}$ corresponden al mismo día, y $t_{3}$ y $t_{4}$, a otro día diferente. En las Figuras 2.e) y 2.f), se muestran la posición relativa de las parejas de nubes \#1-\#2 y \#3-\#4. En primer lugar, han de alinearse, registrarse y sumarse dos a dos, dando como resultado las nubes que se observan en las Figuras 2.g) y 2.h). Por último, se ha de hacer un registro y unión de estas nubes, cuyo resultado se muestra en la Figura 2.i).

El resultado es una única nube de puntos con zonas de densidad variable. Es decir, habrá, por un lado, zonas que únicamente contengan puntos correspondientes a una única captura 3D y, por otro, en el extremo contrario, zonas en las que coincidan las dos parejas de nubes de puntos solapadas. En las Figuras 3.a) y 3.b), se resalta la zona de solapamiento entre las parejas de nubes \#1-\#2 y \#3-\#4, respectivamente. El solapamiento de las cuatro nubes aparece ilustrado en la Figura 3.c).

Tras esto, se evidencia que, aunque cada nube de puntos tiene puntos muy próximos a otros de las otras nubes en las zonas de solapamiento, no puede hacerse una correspondencia inmediata y unívoca entre esos puntos $\mathrm{y}$, por tanto, no se le pueden asignar el valor de las propiedades en otros instantes de tiempo.

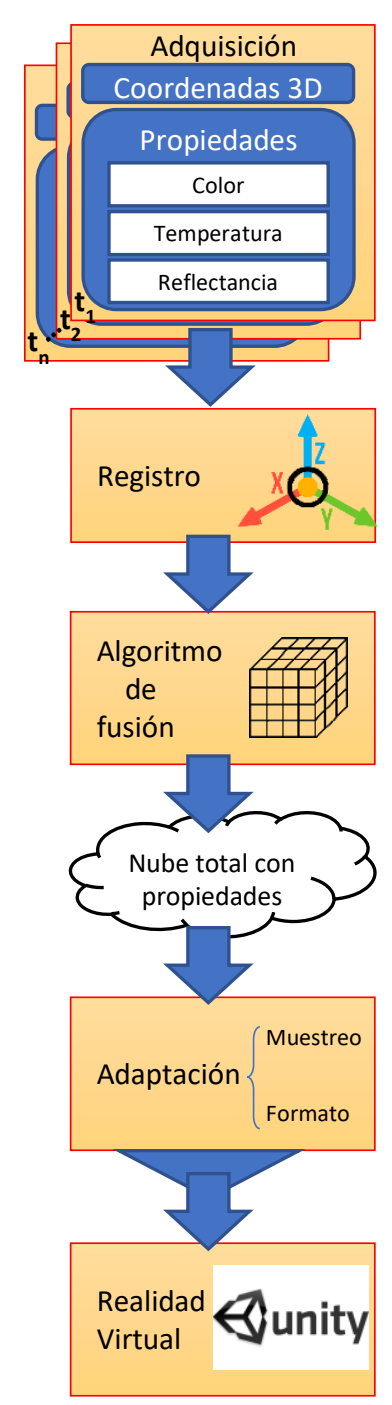

Figura 1: Esquema con las etapas que componen el método desarrollado

Para establecer esa correspondencia que posibilite analizar la evolución de las propiedades en puntos de la nube, se ha creado un algoritmo de fusión. Además de permitir el análisis temporal de la nube, este algoritmo consigue, por un lado, una reducción del número de puntos, algo que se torna esencial para la representación en tiempo real mediante Realidad Virtual. Por otro lado, genera una nube de puntos más 
uniforme, característica que es valorable para la exploración visual de las nubes.

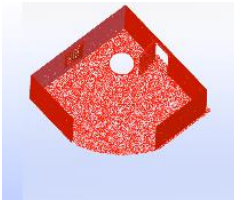

(a)

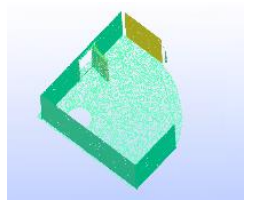

(c)

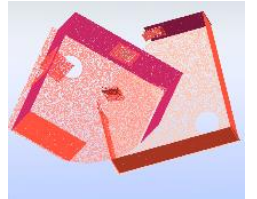

(e)

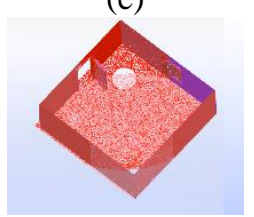

(g)

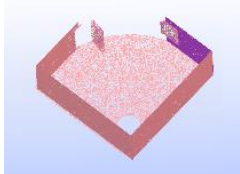

(b)

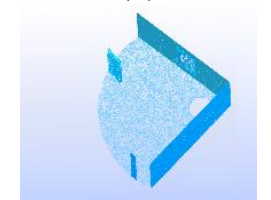

(d)

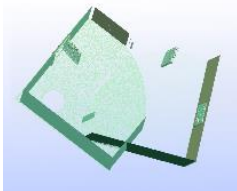

(f)

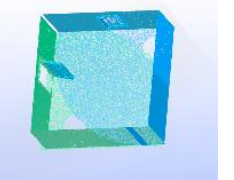

(h)

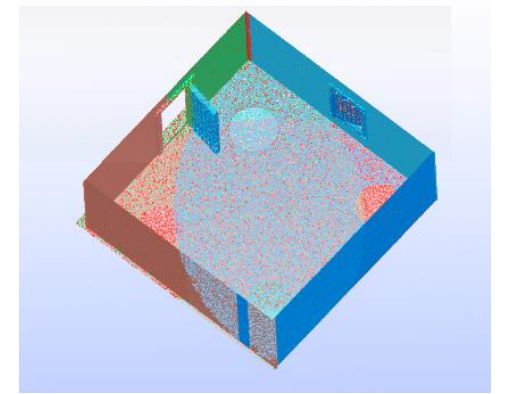

(i)

Figura 2: a) Nube en bruto \#1. b) Nube en bruto \#2. c) Nube en bruto \#3. d) Nube en bruto \#4. e) Nubes en bruto \#1 y \#2. f) Nubes en bruto \#3 y \#4. g) Nubes \#1 y \#2 registradas. h) Nubes \#3 y \#4 registradas. i) Nubes \#1, \#2, \#3 y \#4 registradas.

\subsection{ALGORITMO DE FUSIÓN}

Este algoritmo parte de la unión de las diferentes nubes generadas en la misma habitación (Figura 2.i)), en adelante "nubes de partida". Hay que recordar que cada una de estas nubes consisten básicamente en una lista de coordenadas 3D de cada punto y un valor de la propiedad asociada a cada una de ellas. Antes de llevar a cabo la etapa anterior, es necesario añadir una nueva propiedad, $i$ a cada uno de los puntos de las nubes de partida. Esta propiedad $i$ consiste básicamente en un índice o número entero que referencia la nube de puntos a la que pertenece, o también puede decirse que referencian al mismo periodo de tiempo de adquisición. Por tanto, en el ejemplo de la Figura 2, todos los puntos de las nubes \#1, \#2, \#3 y \#4, tendrán un valor $i$ igual a $1,2,3$ y 4 , respectivamente. De cualquier forma, si la propiedad que se está analizando de la nube de puntos tiene una variación muy lenta, como por ejemplo la temperatura, puede ser útil considerar que nubes adquiridas en instantes de tiempo próximos, comparten el mismo valor $i$. Por tanto, se le asigna el valor de $\mathrm{i}=1$ a las nubes \#1 y \#2 e $\mathrm{i}=2$, a las nubes \#3 y \#4. Una vez ejecutado el registro se tiene una nube total con una estructura como la mostrada en la Figura 4, con los siguientes datos de cada punto:

- Coordenadas X, Y, Z.

- Temperatura en el instante del escaneo.

- Índice que indique a cuál de las nubes de partida pertenecía.

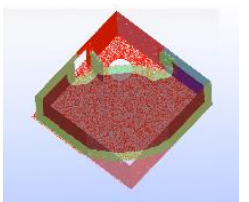

(a)

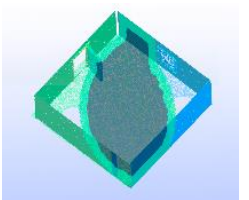

(b)

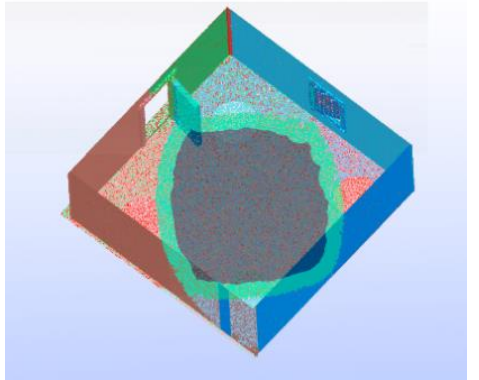

(c)

Figura 3: Solapamiento, resaltado con sombreado entre las diferentes nubes registradas: a) \#1 y \#2; b) $\# 3$ y \#4 c) \#1, \#2, \#3 y \#4.

A continuación, el método propuesto voxeliza la nube de puntos, es decir, divide en regiones iguales el espacio ocupado por la nube de puntos, de forma que los puntos que ocupan un mismo vóxel son finalmente sustituidos por un punto en el centro del mismo. Uno de los parámetros más relevantes en el proceso de fusión es la elección del tamaño del vóxel. Vóxeles grandes consiguen un menor número de puntos en la nube resultado y con información térmica de todos los instantes, pero a su vez reducen la resolución en la representación respecto de las nubes de partida, por este motivo hay que encontrar un punto de equilibrio, que genere un modelo con la información necesaria para una correcta visualización y elimine la redundante. En la Figura 5 se muestra la adaptación de diferentes tamaños de vóxeles a la nube total de la Figura 2.i). La voxelización es una práctica habitual en 
la optimización de nubes de puntos como se aplica, por ejemplo, en [6] y [7].

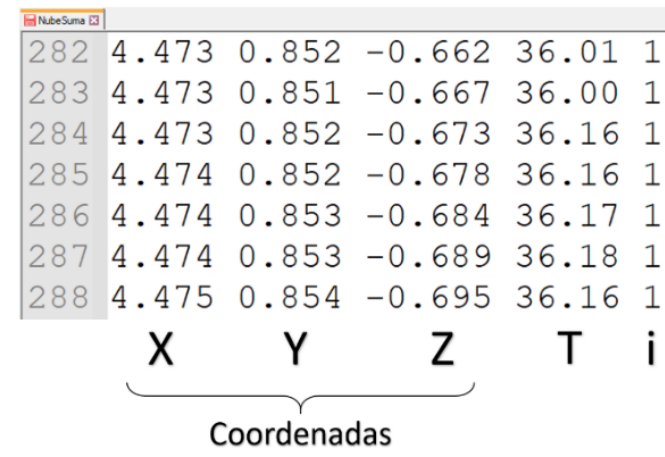

Figura 4: Formato del archivo de la nube que almacena la suma de las nubes en bruto.

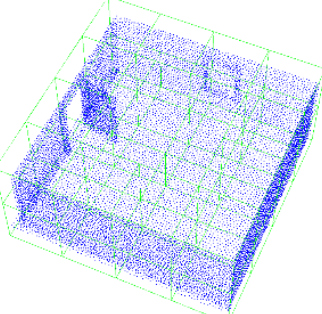

(a)

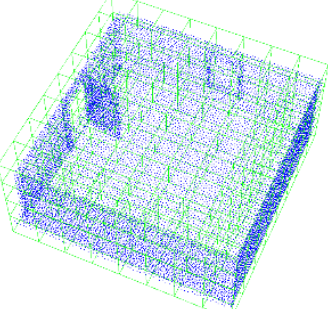

(c)

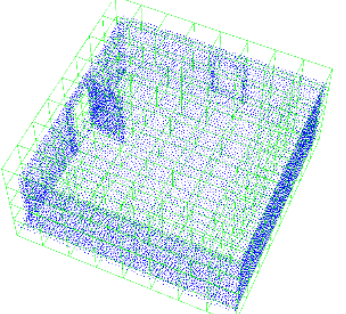

(b)

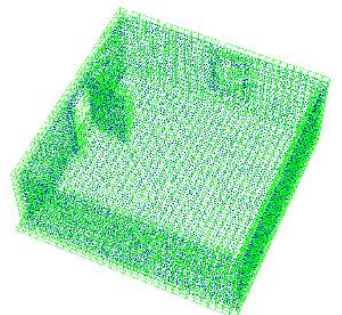

(d)
Figura 5: Densidad de puntos en función del tamaño del vóxel, relación inversa entre el tamaño del vóxel y la cantidad de puntos de la nube. Se han probado diferentes tamaños de vóxeles: a) se corresponde con el de menor tamaño de vóxel y d) con el de mayor tamaño.

Una característica del método planteado es la resolución de la fusión de los puntos pertenecientes a un mismo vóxel, Figura 6, en función de la cantidad de estos. Analizamos a continuación las distintas situaciones que pueden ocurrir:

a. Un solo punto de una de las nubes de partida, en este caso la nube fusionada presentará un punto con información térmica únicamente de ese instante y ninguna de los otros.

b. Un solo punto de cada una de las nubes de partida, el punto representado por este vóxel tendrá información térmica de cada instante igual a la de los puntos de partida.

c. Varios puntos de una o más de las nubes de partida, si un vóxel presenta varios puntos de la misma nube de partida, entonces el punto resultado contendrá como dato térmico de dicho instante la media de las temperaturas de la nube de partida correspondiente.

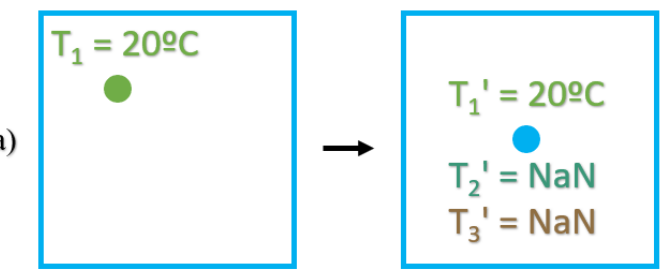

b)
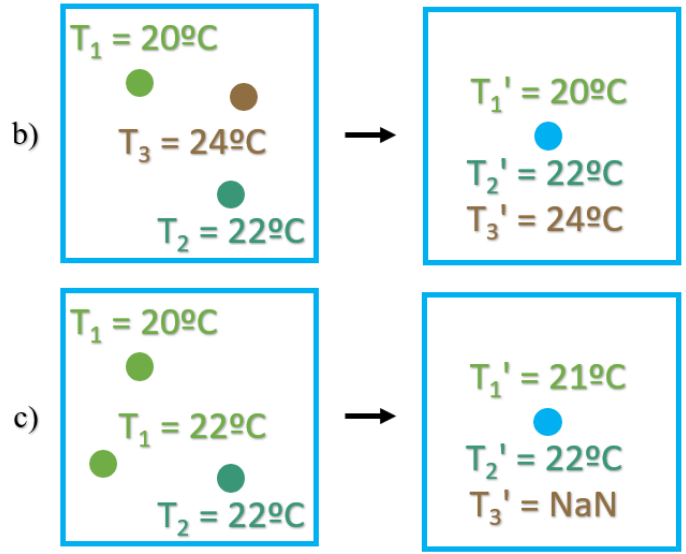

Figura 6: a) Fusión de puntos cuando no se presentan datos en alguna de las nubes. b) Fusión de puntos con información de todas las nubes. c) Fusión de puntos utilizando el valor medio cuando se presentan más de un punto en alguna de las nubes.

Tras el procesamiento de todos los puntos de la nube suma de las nubes de partida se obtiene el archivo resultado, mostrado en la figura 7 , con los siguientes datos de cada punto:

- Coordenadas X, Y, Z.

- Temperaturas en cada instante.

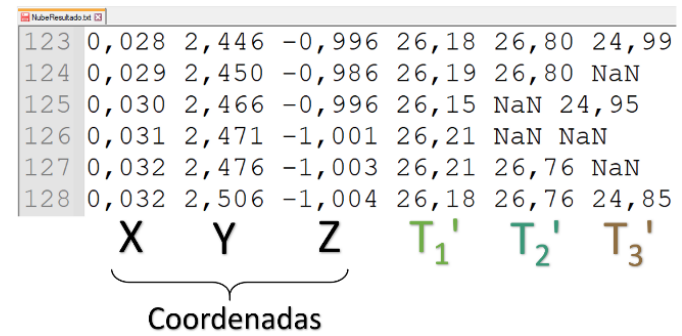

Figura 7: Formato del archivo de la nube fusionada.

El último paso que hay que ejecutar es la adaptación de los datos que serán introducidos en la aplicación de Realidad Virtual. Esta adaptación se lleva a cabo en 
dos etapas. La primera es la etapa de muestreo, en la que se aplica una reducción uniforme del número de puntos, con el objetivo de obtener nubes que no superen los dos millones de puntos aproximadamente. Este valor concreto está determinado por la comprobación empírica de que, con el equipo utilizado (Intel i7, 16 GB RAM, RTX 2060) y, a falta de optimizar la aplicación, nubes de un número de puntos superior a este dan lugar a un funcionamiento poco fluido de la aplicación. La segunda etapa es la de adaptación de formato. En concreto, se ha decidido usar el, cada vez más extendido, formato PLY. Además, se exporta en binario ya que permite unas transferencias de datos (streaming) mucho más rápidas.

\section{APLICACIÓN EN REALIDAD VIRTUAL PARA EXPLORACIÓN DE LA NUBE FUSIONADA}

El objetivo de la aplicación de Realidad Virtual que se ha desarrollado es explorar la nube de puntos de forma interactiva, permitiendo al usuario acceder a toda la información térmica temporal contenida en los puntos, de forma intuitiva y visual, así como cambiar la escala de color de la representación, como se muestra en la Figura 8.

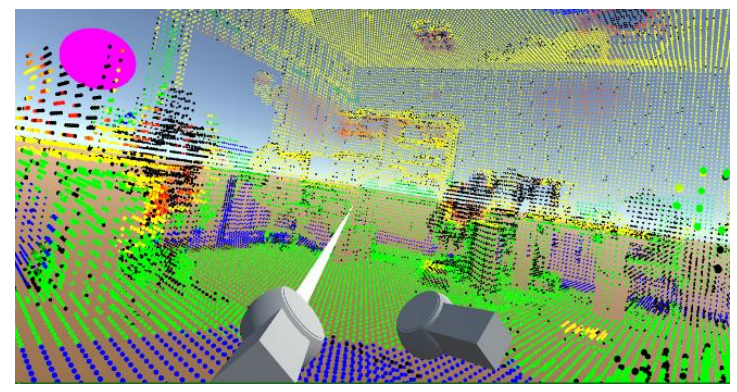

Figura 8: Captura de la aplicación de Realidad Virtual en la se está explorando una nube de puntos y se visualiza en una escala de color el valor de una de sus propiedades.

Esta aplicación ha sido desarrollada mediante el ampliamente extendido motor de tiempo real Unity. Como la gran mayoría de estos motores, Unity está enfocado al uso de modelos 3D compuestos por mallas. Esto es, Unity no soporta de forma nativa el uso de nubes de puntos. Por ello, uno de los pasos fundamentales será la implementación de un módulo que permita superar este inconveniente. La aplicación final se estructura según el esquema que puede observarse en la Figura 9.

Para trabajar con las nubes de puntos en Unity se ha implementado un importador que parte del archivo PLY y convierte la nube en un GameObject con ciertos componentes. Los GameObject son los elementos fundamentales con los que Unity compone las escenas y una de cuyas características principales es el componente para su transformación (Transform) en el mundo 3D: posicionado, rotación y escalado. Además, internamente se crea un vector de puntos, Punto[i], cuyo elemento fundamental es la estructura Punto, compuesta por tres componentes: Vector de posición con las coordenadas $x, y, z$; valor de la propiedad; e índice temporal.

Por otro lado, es primordial crear, a partir del vector de puntos, un árbol octree, para tener almacenados los datos en una estructura espacial ordenada.

Como se ha indicado previamente, Unity no integra un mecanismo propio para la representación o renderizado de nubes de puntos, por lo que se requiere del desarrollo de una estructura de datos, Punto_r ri], compuesta por las coordenadas y el color (RGB) del punto, que la tarjeta gráfica procesa y envía para ser representados. Por tanto, para la representación de los puntos es necesario un mecanismo para transformar los datos de la estructura Punto[i] a la estructura Punto_r[i]. Para ello, se hace una conversión del valor de la propiedad a una escala de color creciente AzulVerde-Amarillo-Rojo, en donde, el Azul se corresponde con los valores más bajos y el Rojo con los valores más altos de la propiedad. En este paso, se le da al usuario la posibilidad de escalar la representación en color, es decir, de elegir qué valor mínimo de la propiedad se asigna al mínimo de la escala de color y qué valor máximo al máximo de color.

Al GameObject de la nube de puntos que se quiere explorar con la aplicación hay que añadirle varios componentes (scripts) que otorgan funcionalidades para una mejor representación de la información. Entre las diferentes funcionalidades destacan:

- Cambio de tamaño de los puntos.

- Cambio de las escalas de colores.

- Elección del instante del cual se quiere visualizar la información.

- Señalización del punto con el valor más elevado de temperatura.

- Sondeo de los puntos para obtener información del valor de temperatura (Figura 11).

Dentro de las funcionalidades tratadas, algunas hacen uso de un menú, que puede verse en la Figura 10, para cambiar las propiedades y configurar la nube de acuerdo a las preferencias del usuario.

El sondeo de valores de las propiedades dentro de la nube de puntos es posible realizarlo en tiempo real gracias a la consulta dentro de la estructura de árbol 
octree, que optimiza y acelera enormemente las búsquedas dentro del enorme conjunto de datos que constituye la nube de puntos. Otros trabajos, como el mostrado en [8] demuestran las ventajas del uso de estas técnicas.

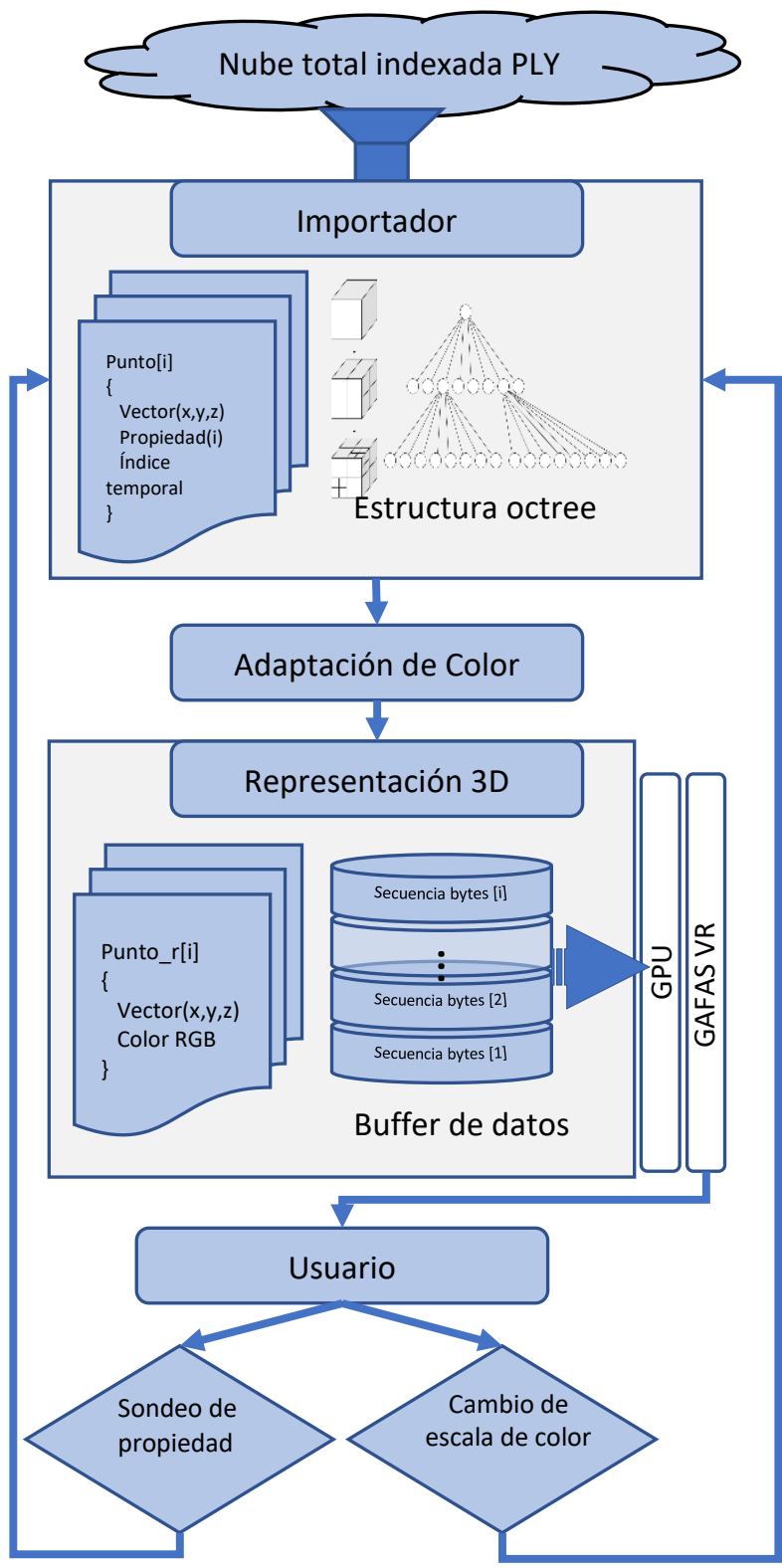

Figura 9: Estructura aplicación Realidad Virtual

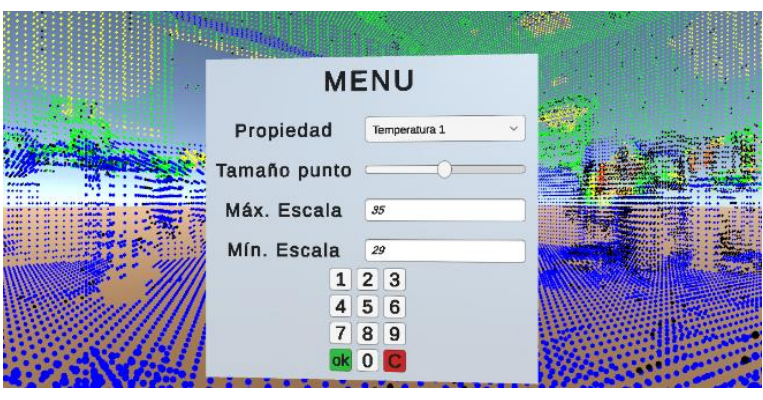

Figura 10: Interfaz de usuario de la aplicación en la que puede elegirse la propiedad a representar, el tamaño del punto, y la variación de los límites de la escala de color.

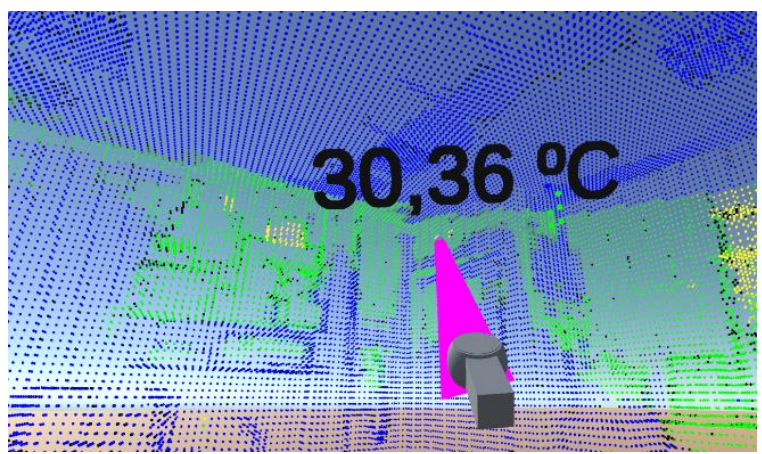

Figura 11: Sondeo de la temperatura de un punto de la nube que se está explorando.

En cuando a los resultados experimentales, específicamente, se han realizado pruebas con un conjunto de nubes de puntos obtenidas por un escáner 3D RieglVz 400 el cual tenía incorporada una cámara térmica, FLIR AX5, que permite asociar información térmica a cada punto de la nube.

\section{CONCLUSIONES}

El sistema descrito ha resultado funcional $y$ ha cumplido con los objetivos de reducción de la información irrelevante conservando los datos que varían en el tiempo. Además, la experiencia de navegación es fluida y visual, permitiendo captar de forma rápida las diferentes temperaturas de las zonas registradas. La dificultad de procesamiento se ve reducida notablemente ya que se pasa de varías nubes a una, haciendo accesible su uso en sistemas que precisen de optimización como el de la aplicación de Realidad Virtual que ha sido desarrollada.

En el futuro sería interesante extender las pruebas realizadas a otras propiedades que varíen en el tiempo. Por ejemplo, podría resultar de interés la variación del valor de las sombras para estudios de generación fotovoltaica. También asociado a la producción de energía por radiación solar podría ser valioso la 
creación de nubes de puntos con información de la variación de la irradiación.

Dentro de la optimización de la aplicación de navegación de las nubes caben más mejoras como la renderización únicamente de los elementos que se encuentre dentro del campo de visión (frustrum culling).

\section{Agradecimientos}

Este trabajo ha sido financiado por el proyecto titulado "Procesamiento de datos masivos de escáneres 3D: Segmentación, reconocimiento y modelado geométrico de edificaciones no convencionales", con referencia AEI/PID2019-108271RB-C32/ 10.13039/501100011033; y el proyecto "Tecnologías 3D para la resiliencia, la recuperación y la sostenibilidad del Patrimonio Cultural Extremeño", con referencia IB20172, de la Junta de Extremadura y el Fondo Europeo de Desarrollo Regional "Una manera de hacer Europa".

\section{English summary}

\section{POINT CLOUD TIME SERIES FUSION FOR VIRTUAL REALITY EXPLORATION}

\begin{abstract}
The amount of information that can be shown in point clouds is increasing. Part of this information changes over time. For this reason, is interesting to develop a system that merges data of several clouds into a single cloud which maintains the variable data and remove redundancy in spatial information. Furthermore, it is valuable for system that are dependent on optimization as Virtual Reality system. In this paper the method that generates the point cloud with all the necessary information will be described. Finally, the results will be tested in a Virtual Reality app developed for this purpose. The app will permit to explore to point cloud and to check the values of the points.
\end{abstract}

Keywords: Point clouds, Virtual Reality, thermal information, information optimization.

\section{Referencias}

[1] Adán, A., Quintana, B., Aguilar, J.G., Pérez, V., Castilla, F.J., (2020) Towards the use of 3D thermal models in constructions, Sustain. 12 1-13.

[2] Besl, P.J., McKay, N.D., (1992) A method for registration of 3-D shapes, IEEE Trans. Pattern Anal. Mach. Intell. 14 239-256.

[3] Elseberg, J., Borrmann, D., Nüchter, A., (2013) One billion points in the cloud - An octree for efficient processing of 3D laser scans, ISPRS J. Photogramm. Remote Sens. $7676-88$.

[4] Montilla, M., Orjuela-Vargas, S.A., Philips, W., SPIE, 2014State of the art of 3D scanning systems and inspection of textile surfaces, in: Meas. Model. Reprod. Mater. Appear., : p. 90180A.

[5] Özbay, E., Çinar, A., (2019) A voxelize structured refinement method for registration of point clouds from Kinect sensors, Eng. Sci. Technol. an Int. J. 22 555-568.

[6] Pomerleau, F., Colas, F., Siegwart, R., Magnenat, S., (2013) Comparing ICP variants on real-world data sets: Open-source library and experimental protocol, Auton. Robots. 34 133-148.

[7] Quan, S., Ma, J., Hu, F., Fang, B., Ma, T., (2018) Local voxelized structure for 3D binary feature representation and robust registration of point clouds from low-cost sensors, Inf. Sci. (Ny). 444 153-171.

[8] Virtanen, J.-P., Daniel, S., Turppa, T., Zhu, L., Julin, A., Hyyppä, H., Нyyppä, J., (2020) Interactive dense point clouds in a game engine, ISPRS J. Photogramm. Remote Sens. 163.

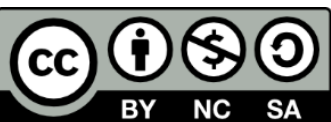

(C) 2021 by the authors. Submitted for possible open access publication under the terms and conditions of the Creative Commons Attribution CC BY-NC-SA 4.0 license (https://creativecommons.org/licenses/byncsa/4.0/deed.es). 